\title{
Optical Sensing of Attached Fibrinogen on Carbon Doped Titanium Surfaces
}

\author{
Raimo Silvennoinen, ${ }^{1}$ Vladimír Vetterl, ${ }^{2}$ Stanislav Hasoň, ${ }^{2}$ Martti Silvennoinen, ${ }^{1}$ \\ Kari Myller, ${ }^{3}$ Jiři Vaněk, ${ }^{4}$ and Ladislav Cvrček ${ }^{5}$ \\ ${ }^{1}$ Department of Physics and Mathematics, University of Eastern Finland, Joensuu Campus, P.O. Box 111, 80101 Joensuu, Finland \\ ${ }^{2}$ Institute of Biophysics, v.v.i., Academy of Sciences of the Czech Republic, Královiopolská 135, 61265 Brno, Czech Republic \\ ${ }^{3}$ MGM-Devices Ltd., 82210 Suhmura, Finland \\ ${ }^{4}$ Centre for Dental and Craniofacial Research, Faculty of Medicine, Masaryk University, Komenského nám 2, \\ 66243 Brno, Czech Republic \\ ${ }^{5}$ HVM Plasma Ltd., Research and Development Department, Na Hutmance 2, 15800 Prague 5, Czech Republic
}

Correspondence should be addressed to Raimo Silvennoinen, raimo.silvennoinen@uef.fi

Received 24 November 2009; Accepted 2 March 2010

Academic Editor: Peter V. Polyanskii

Copyright (C) 2010 Raimo Silvennoinen et al. This is an open access article distributed under the Creative Commons Attribution License, which permits unrestricted use, distribution, and reproduction in any medium, provided the original work is properly cited.

The adsorption/desorption of Human Plasma fibrinogen (HPF) molecules on biosurfaces was measured in spectroscopic cuvette by a diffractive optical element- (DOE-) based sensor. To characterize the surfaces, the basic parameters as surface tension was obtained by sensing of a contact angle of water droplet and dielectric constant was measured by ellipsometry in the absence of HPF molecules. It was observed a significant correlation between the adsorption ability of HPF molecules (sensed by DOE on the basis of the changes in optical roughness $\left(R_{\text {opt }}\right)$ of studied surface in the absence and presence of HPF molecules), and dielectric constant (measured by ellipsometry) of differently treated titanium surfaces, where the permittivity and dielectric loss have the known linear relation. These findings with carbon-treated biomaterial surfaces can help us to understand mechanisms behind attachment of HPF molecules on biomaterial surfaces to realize and extend variety of implants for hard tissue replacement.

\section{Introduction}

Titanium is frequently used as a biomaterial for hard tissue replacement, such as dental and orthopaedic implants, and biomaterial devices made of titanium give a satisfactory performance [1-7]. The effective surface energy related to topography of surface, which can be varied by different processing methods, is assumed to influence to the final interactions of the implant with the surrounding environment. Rough surfaces promote better osseointegration than smooth surfaces [8-11]. Within a few seconds after implantation the biomaterial surface becomes coated with a film of adsorbed proteins, which mediate the interaction between the implant and the body environment. Since most implants are exposed to blood during implantation, the initial protein film is mainly composed of plasma proteins. Human plasma fibrinogen (HPF) is one of the most relevant proteins adsorbed on biomaterial surfaces. HPF partakes in blood coagulation facilitate adhesion and aggregation of platelets $[12,13]$. The structure and composition of the adsorbed protein layer determine the type and extent of the subsequent biological reactions, such as activation of coagulation and immune response and osseointegration [14]. The initially adsorbed protein layer is thus a factor for conditioning the biocompatibility [15-17]. The mechanisms and the factors, which are important for protein adsorption and desorption, are still subject of scientific research and not understood very well. Therefore it is important to investigate how different surfaces influence the formation and properties of adsorbed protein layers.

In this paper we express characteristics, which relate to the adsorption/desorption of HPF molecules on differently treated titanium surfaces. The treatments were (i) mechanical polishing and (ii) plasma-enhanced chemical 


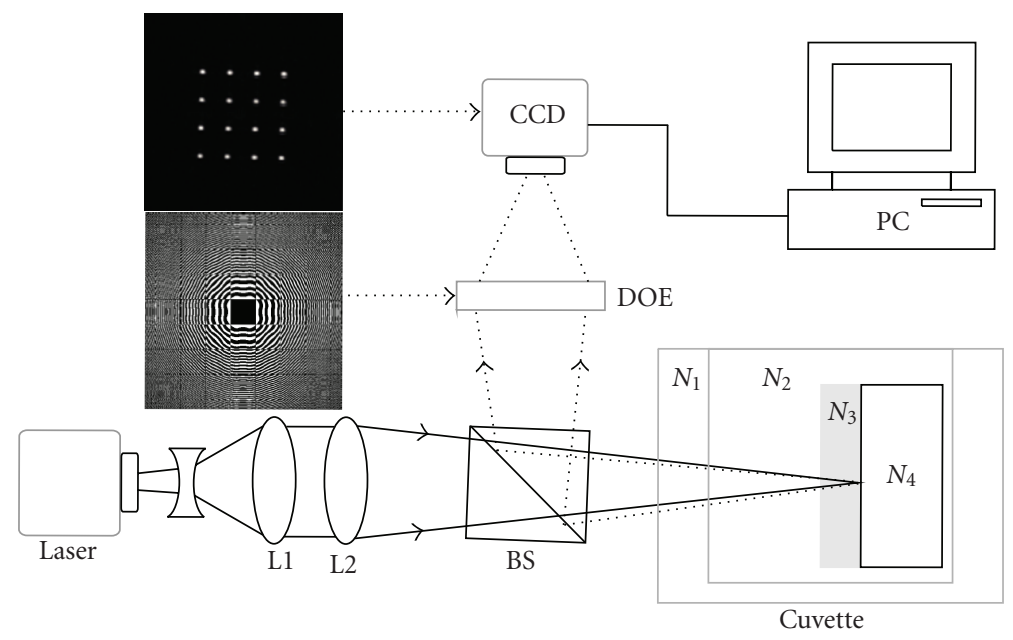

FIGURE 1: Geometric setup of DOE sensor with sample cuvette compartment for $R_{\text {opt }}$ measurements. Lower inset denotes aperture of DOE whereas upper inset denotes reconstructed $4 \times 4$ spot matrix image of DOE by using nondistorted wavefront.

vapor deposition (PECVD) of either titanium carbide layers with different concentrations of carbon (three samples) or diamond-like carbon (DLC) coating. The surface tension and surface energy of the samples were obtained from optical measuring of the contact angle of distilled water droplet on the dry bulk sample surface. The dielectric constant of each bulk surface was measured in dry environment utilizing ellipsometry. The temporal adsorption process of HPF molecules on test surfaces was measured in vitro using one arm optical interferometer, which utilizes a diffractive optical element (DOE) $[18,19]$. This optical interferometer works in coherent and in noncoherent mode, which allows sensing of optical path differences providing information on the optical roughness $\left(R_{\mathrm{opt}}\right)$, and reflectance of the surfaces immersed into various liquids. This method can thus be used for the study of the interactions of the molecules dissolved in the liquid with the surface to find out parameters to understand mechanisms behind adsorption/desorption of HPF molecules on biomaterial surfaces to realize implants in hard tissue replacement.

\section{Materials and Methods}

\subsection{Ellipsometry and Water Contact Angle of Treated Titanium} Surfaces. In this work were used following surfaces: (1) mechanically polished titanium, (2)-(4) plasma-enhanced chemical vapor deposited titanium carbides with three different concentrations of carbon $\left[\mathrm{Ti}_{0.82}-\mathrm{C}_{0.18}(2)\right.$; $\mathrm{Ti}_{0.38}-$ $\mathrm{C}_{0.62}$ (3); $\mathrm{Ti}_{0.09}-\mathrm{C}_{0.91}$ (4)], and (5) diamond-like carbon $\left(\mathrm{Ti}_{0.00}-\mathrm{C}_{1.00}\right)$. The thickness of titanium oxide layer was measured with polished titanium surface, and its depth was about $220 \mathrm{~nm}$. The thickness of $\mathrm{Ti}_{x}-\mathrm{C}_{1-x}$ coatings produced by using PECVD ranged from $2.5 \mu \mathrm{m}$ to $3.5 \mu \mathrm{m}$, which is thick enough in optical sense to consider it as solid bulk layer $[20,21]$. The ellipsometric measurements of these differently treated surfaces were performed in dry environment to gain information about the permittivity possible related to adsorption of HPF molecules. The complex refractive index

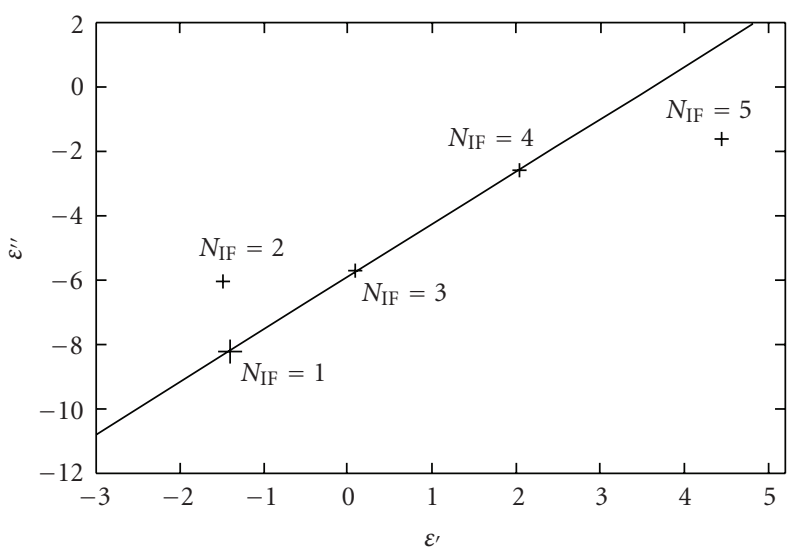

FIgUre 2: Complex effective dielectric constants (dielectric permittivity) for treated titanium surfaces at $E_{\lambda}=1.959 \mathrm{eV} . N_{\mathrm{IF}}=1 \equiv$ polished titanium, $N_{\mathrm{IF}}=2 \equiv \mathrm{Ti}_{0.82}-\mathrm{C}_{0.18}, N_{\mathrm{IF}}=3 \equiv \mathrm{Ti}_{0.38}-\mathrm{C}_{0.62}$, $N_{\mathrm{IF}}=4 \equiv \mathrm{Ti}_{0.09}-\mathrm{C}_{0.91}$, and $N_{\mathrm{IF}}=5 \equiv \mathrm{Ti}_{0.00}-\mathrm{C}_{1.00}$ (diamond-like carbon). The standard deviations in permittivity $\varepsilon^{\prime}$ and dielectric loss $\varepsilon^{\prime \prime}$ directions are indicated on each dielectric constant by horizontal and vertical lines. Parameters for the line are as follows: $\varepsilon^{\prime \prime}=a \varepsilon^{\prime}+b$, where $a=1.634$ and $b=-5.877$.

values $\left(N_{n \kappa}=n-i \kappa\right)$, in turn, were utilized in the calculation of the effective dielectric constant $\varepsilon=\varepsilon^{\prime}+i \varepsilon^{\prime \prime}=N_{n \kappa}^{2}$, where $\varepsilon^{\prime}=n^{2}-\kappa^{2}$ and $\varepsilon^{\prime \prime}=-2 n \kappa$. To avoid the harmful effects caused by the possible appearance of surface roughness [22, 23], the ellipsometric measurements were performed at the incidence angle of $75^{\circ}$ for probe beam utilizing Woollam variable angle spectroellipsometer (W-VASE), which is operating in the wavelength range from $200 \mathrm{~nm}$ to $1700 \mathrm{~nm}$. The complex permittivity values of the all studied surfaces were calculated from the complex refractive index values, which were obtained by the spectroellipsometer (cf. Table 1). The surface energy of each of studied surfaces was estimated from optical measurements of the contact-angle of water droplet injected on the dry surface (cf. Table 1). 
TABle 1: Complex effective dielectric constants (dielectric permittivity) $\varepsilon=\varepsilon^{\prime}+i \varepsilon^{\prime \prime}$ at $E_{\lambda}=1.959 \mathrm{eV}$ with standard deviations and water contact angles $\left(\theta^{\circ}\right)$ for the five studied surfaces. Sample number indexes $N_{\text {IF }}$ shown in Figure 2 are listed according to severity of dielectric losses $\varepsilon^{\prime \prime}$.

\begin{tabular}{lcccc}
\hline Sample & Treatment & $\varepsilon$ & $\Delta \varepsilon\left(\times 10^{-2}\right)$ & $\theta^{\circ}$ \\
\hline 1 & Polished titanium & $-1.406-8.207 i$ & $34.1+128.4 i$ & $66.5 \pm 4.1$ \\
2 & PECVD Ti $_{0.82}-\mathrm{C}_{0.18}$ & $-1.492-6.024 i$ & $17.2+23.1 i$ & $81.1 \pm 2.6$ \\
3 & PECVD Ti $_{0.38}-\mathrm{C}_{0.62}$ & $0.084-5.681 i$ & $20.5+40.4 i$ & $77.9 \pm 3.1$ \\
4 & PECVD Ti $_{0.09}-\mathrm{C}_{0.91}$ & $2.034-2.580 i$ & $6.7+3.4 i$ & $75.0 \pm 0.4$ \\
5 & DLC Ti $_{0.00}-\mathrm{C}_{1.00}$ & $4.448-1.570 i$ & $13.5+14.9 i$ & $70.7 \pm 2.0$ \\
\hline
\end{tabular}

2.2. Coherent Response of DOE Sensor. The thicknesses of the adsorbed layers of HPF molecules on differently treated titanium surfaces were sensed utilizing the coherence response of DOE sensor as shown in Figure 1. The DOE sensor uses expanded and focused laser beam $(\lambda=633 \mathrm{~nm})$ realized with the aid of the lens system L1-L2 to hit on studied surface $\mathrm{N}_{4}$ trough reference liquid (water) in cuvette via beam splitter BS and cuvette window $N_{1}$. Backscattered laser beam is directed with the aid of beam splitter on DOE aperture (shown in the lower inset of Figure 1), which analyses if the wavefront is distorted after adsorption either the ions of background electrolyte or added HPF molecules (denoted as $N_{3}$ ) on studied surface in the aqueous environment of background electrolyte $\left(N_{2}\right)$. Distorted $4 \times 4$ light spot DOE image is grabbed from two-dimensional (2D) photo array of the charge-coupled device (CCD) and analyzed using a personal computer (PC). The changes in $R_{\mathrm{opt}}$, which relate to surface roughness $R_{\mathrm{a}}$ [24], are detected utilizing the coherent response of the DOE sensor. The thickness of the adsorbed layer on treated titanium surfaces is calculated from the captured DOE image data of the $4 \times 4$ light spot matrix, which is shown in the upper inset of Figure 1. The irradiance of the peaks was calculated utilizing (1) as follows:

$$
I_{C}=\frac{1}{n_{p k} m_{p k}} \sum_{i_{p k}=1, j_{p k}=1}^{n_{p k}, m_{p k}} I_{i_{p k}, j_{p k}},
$$

where $n_{p k}$ and $m_{p k}$ are the pixel dimensions of each 16 peaks in DOE image and $I_{i_{p k}, j_{p k}}$ is the image irradiance observed by the $\left(i_{p k}, j_{p k}\right)$ th element of the peak in DOE image captured by a CCD camera. The 16 different diffractive lenses are integrated utilizing superposition principle in the DOE aperture obeying coherent response for each pixel with complex wavefront amplitude $A_{i, j}$ as follows: $I_{C}=\left|\sum A_{i, j}\right|^{2}$, which satisfies the principle of compact and phase sensitive interferometer. The DOE element images the $4 \times 4$ light spot matrix in its focal plane. If the reconstructing wavefront does not satisfy the terms of hologram imaginary, the spot image matrix does not appear in the image plane. The same holds, for instance, in the case, where the radiant exitance from the laser resonator in $\mathrm{TEM}_{00}$ mode starts to suffer from appearance of side modes, and DOE will spatially filter out those images from its original $4 \times 4$ light spot image. With the tedious numerical simulations, it is showed that the irradiance of the $4 \times 4$ spots will decrease as a function of optical path length (OPL) and disappears when the OPL exceeds $\lambda / 4$. This response is published and appears in Figure 8.21(b) of [18]. It is also observable that this response resembles the response of Beckmann-Spizzichino model [25]. To discover the thickness of the adsorbed layer $\mathrm{N}_{3}$ we first calculated the irradiance of the peaks utilizing (1) and after that the optical path difference $\Delta r$, understood as an optical roughness $\left(R_{\text {opt }}\right)$, is solved inversely by using this response. We have noted during our previous measurements that the accuracy of $0.2 \mathrm{~nm}$ can be achieved by using this one arm interferometric technique [19]. The similar accuracy limits are also reported recently for the coupling dynamics of lasers of self-mixing interferometers in vibrometer applications ranging from $0.1 \mathrm{~nm}$ to $100 \mu \mathrm{m}$ [26]; whereas the accuracy of conventional two arm interferometers used in optical diagnostics of random phase objects [27] as well as in optical diagnostics of rough surfaces [28] is estimated to be $\sim 0.005 \mu \mathrm{m}$.

\subsection{DOE Sensor Measurements of Treated Titanium Surfaces.} First the DOE sensor images were made in water for 100 seconds in aim to perform the reference signal level from each surface, and during that time frame 1000 reference samples were grabbed. Thereafter the water was removed by syringe from cuvette and the background electrolyte was injected in the cuvette. Immediately after injection of background electrolyte, the grabbing of the DOE images was started, and the image grabbing was repeated after two minutes interval. Before HPF measurement, the cuvette was washed, and after washing the new treated titanium sample was installed in the sample holder inside the cuvette. The water was injected in the cuvette, and the DOE image references from the new sample surface were taken. Before adding the HPF solution in the cuvette, the immersion water was removed, and DOE image grabbing process was started. The image grabbing was repeated two times consecutively after two minutes interval. The diameter of the laser beam waist on the all surfaces was $1 \mathrm{~mm}$. Thereafter we compared the optical roughness $R_{\text {opt }}$ values, which were measured by DOE sensor as a function of time from the interface of the treated titanium surface-electrolyte in the absence or presence of HPF molecules. The threshold of optical roughness of the treated titanium surface was cancelled out by measuring the base line of $R_{\mathrm{opt}}$ in distilled water, which refractive index $(n=1.333)$ was close to background electrolyte $(n=1.338)$. The $R_{\text {opt }}$ values for all studied surfaces in the absence and presence of HPF molecules are shown in Table 2. 
TABLE 2: Optical roughness $\left(R_{\text {opt }}\right)$ of the five studied surfaces with standard deviations at $E_{\lambda}=1.959 \mathrm{eV}$ immersed in background electrolyte in absence or presence of HPF molecules. Sample number indexes $N_{\text {IF }}$ resemble those appearing in Figure 2.

\begin{tabular}{|c|c|c|c|c|}
\hline \multirow{2}{*}{ Sample $N_{\mathrm{IF}}$} & \multicolumn{2}{|c|}{ Absence of HPF molecules } & \multicolumn{2}{|c|}{ Presence of HPF molecules } \\
\hline & $R_{\text {opt }}(\mathrm{nm})$ & $\Delta R_{\mathrm{opt}}(\mathrm{nm})$ & $R_{\mathrm{opt}}(\mathrm{nm})$ & $\Delta R_{\text {opt }}(\mathrm{nm})$ \\
\hline 1 & 23.2 & 3.2 & 39.2 & 9.7 \\
\hline 2 & 25.7 & 9.1 & 17.4 & 6.7 \\
\hline 3 & 10.7 & 5.0 & 25.0 & 7.1 \\
\hline 4 & 11.4 & 4.6 & 27.2 & 7.5 \\
\hline 5 & 35.5 & 2.6 & 29.9 & 6.7 \\
\hline
\end{tabular}

2.4. Chemicals. Human plasma fibrinogen (HPF), fraction I, type III were purchased from Sigma. In all experiments the HPF was dissolved in phosphate buffer solution (PBS) $+0.1362 \mathrm{M}$ sodium citrate, which serve as a background electrolyte at a concentration of $500 \mathrm{nM}$. Measurements were performed at room temperature.

\section{Results and Discussion}

In Figure 2 are shown the effective dielectric constants (dielectric permittivity) from five studied surfaces at photon energy $E_{\lambda}=1.959 \mathrm{eV}$. The first sample represents mechanically polished titanium surface, which act as the reference $\left(N_{\mathrm{IF}}=1\right)$. The three titanium carbide and diamond-like carbon samples were produced PECVD method controlling the severity of chemical vapor deposition in plasmaenhancement to satisfy the carbon concentrations and are denoted as follows: $N_{\mathrm{IF}}=2-5$. The standard deviations in permittivity $\varepsilon^{\prime}$ and dielectric loss $\varepsilon^{\prime \prime}$ directions, which are shown in Table 1, are indicated on each dielectric constant by horizontal and vertical lines in Figure 2.

The optical roughness $\left(R_{\mathrm{opt}}\right)$ values for each of the studied surfaces in the absence or presence of the HPF molecules were calculated from DOE sensor measurements performing under wet environment (Table 2). The $R_{\text {opt }}$ data reveals that the adsorption of HPF molecules is significant for the three surfaces $\left(N_{\mathrm{IF}}=1, N_{\mathrm{IF}}=3\right.$ and $\left.N_{\mathrm{IF}}=4\right)$ compared with the other two surfaces $\left(N_{\mathrm{IF}}=2\right.$ and $N_{\mathrm{IF}}=$ 5 ). Let us denote later these two set of surfaces by $A$ and $B$, respectively. In the deeper evaluation it was observed that in the permittivity $\varepsilon^{\prime}$ and dielectric loss $\varepsilon^{\prime \prime}$ plane the $\left(\varepsilon^{\prime}, \varepsilon^{\prime \prime}\right)$-point value representing each surface in set $\mathrm{A}$ hit on a line, which is also shown in Figure 2; whereas the other two points with the coordinate pairs of set $B$ (including titanium carbide surface $N_{\mathrm{IF}}=2$ and diamond-like carbon $N_{\text {IF }}=5$ surface) do not coincident the line. This notation allows us to make an assumption that the adsorption of HPF molecules relates to a slope of dielectric loss and permittivity $\left(\partial \varepsilon^{\prime \prime} / \partial \varepsilon^{\prime}\right)$ as follows: $\varepsilon^{\prime \prime}=a \varepsilon^{\prime}+b$, where $a=1.634$ and $b=-5.877$. Also the distance of $\left(\varepsilon^{\prime}, \varepsilon^{\prime \prime}\right)$-point from the line $\varepsilon^{\prime \prime}=1.634 \varepsilon^{\prime}-5.877$ indicates that the surfaces in the set A have small distance deviation $(0.017,0.036$, and $0.013)$, whereas in the set $B$ the respective distances deviate more than one decade being (1.198 and 1.547). To compare furthermore permittivity values of the five test surfaces to those surfaces, which are considered to have toxic effects on some bacteria, viruses, and other microbial organisms in vitro, as silver, mercury, and germanium [29-31], we calculated those permittivity values at photon energy $E_{\lambda}=$ $1.959 \mathrm{eV}$ from complex refractive index values available in the series of books of Palik [32]. It is worth to observe that the permittivity values for silver, mercury and germanium, which are $\varepsilon_{\mathrm{Ag}}=-16.174-1.093 i, \varepsilon_{\mathrm{Hg}}=29.257-$ $8.916 i$ and $\varepsilon_{\mathrm{Ge}}=-23.443-20.715 i$, deviate significantly from the permittivity values of the five test surfaces. This can be concluded from the respective distance values, which are as follows: 16.255, 12.109 and 26.492 being rather huge compared with deviating distances in the surface set A. In Figure 3 is shown two SEM images. The both images are from the set A to show the different surface morphology. The polished titanium surface ( $\operatorname{set} A-N_{\mathrm{IF}}=1$ ) looks rather uniform containing some grooves, which is assumed to arise from the polishing process. The titanium carbide surface with lower content of titanium ( set $A-N_{\mathrm{IF}}=4$ ) consists of nanometre scale carbide agglomerates, which in turn is assumed to be originated from the surface energy driven grain growth [33].

The water contact angles $(\theta)$ of the surfaces, those appear in Figure 3, indicate that the surfaces are hydrophilic with level ranging from $81^{\circ}\left(\sim 0.100 \mathrm{~J} / \mathrm{m}^{2}\right)$ to $66^{\circ}\left(\sim 0.173 \mathrm{~J} / \mathrm{m}^{2}\right)$, see Table 1. However estimated from water contact-angles the surface energy density $(\gamma)$ of the titanium carbide surfaces covered by nanometre-scale agglomerates $(\theta$ is decreasing consecutively from $N_{\mathrm{IF}}=2$ to $N_{\mathrm{IF}}=5$ ) are smaller than the surface energy density of carbon black $\left(0.257 \mathrm{~J} / \mathrm{m}^{2}\right)$ in comparison to that of the same carbon black after graphitization $\left(0.189 \mathrm{~J} / \mathrm{m}^{2}\right)$ [34] and the carbon nanofibers surface [35]. The difference should originate from the agglomerates, which decrease the effective contact area of water droplet and titanium carbide increasing the effective area of gas-water interface under droplet. Although the surface energy density does not correlate direct, one to one, with the ability of the adhesion of HPF molecules on surfaces nevertheless it have influences to the hydrophilic interaction of background electrolyte and surface. Moreover, the measured contact angle of water droplet and electrolyte on dry surfaces did not differed significantly from each other whereas the contact angle of electrolyte droplet with HPF molecules was ca. 10 per cent lower than the respective contact angles of water and electrolyte. On the contrary, the hydrophobic behaviour may contribute appearance of 


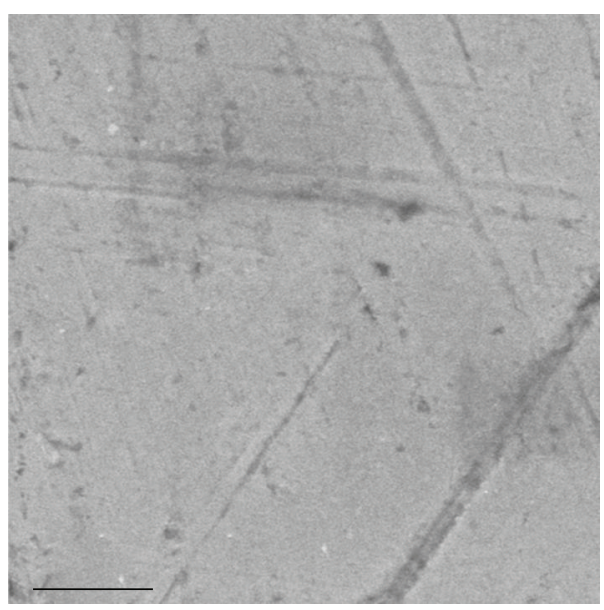

(a)

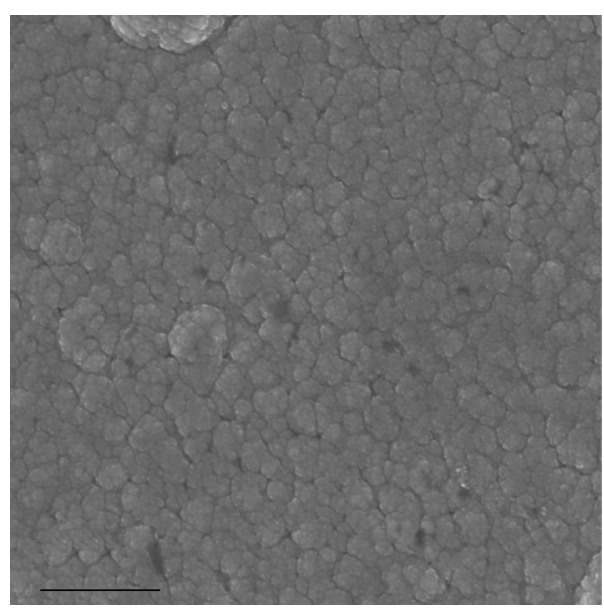

(b)

FIGURE 3: SEM image from two different treated titanium surface: (a) $N_{\mathrm{IF}}=1 \equiv$ polished titanium and (b) $N_{\mathrm{IF}}=3 \equiv \mathrm{Ti}_{0.38}-\mathrm{C}_{0.62}$. The length of black horizontal scale bar in left lower corner of each image is $1 \mu \mathrm{m}$.

nanobubbles at the interface between water (including background electrolyte) and hydrophobic solid surface [36].

The interaction of the probe light with the surfaces is also estimated utilizing the reflectance $R$, which relates to energy loss or absorption of photons. The reflectance $R$ is calculated from the relation $R=\left|\left(1-N_{n \kappa}\right) /\left(1+N_{n \kappa}\right)\right|^{2}$. The reflectance responses of the five studied surfaces were showing decreasing evolution as a function of photon energy in the range from $1.5 \mathrm{eV}$ to $3.0 \mathrm{eV}$ including the energy of probe light $(1.959 \mathrm{eV})$ used in the experiments. The Pearson second-moment correlation of the five measured surfaces between the absorption $(1-R)$ and the dielectric losses $\varepsilon^{\prime \prime}$ at the probe light energy is $r^{2}=0.9313$, which do not explain one to one the ability of the adhesion of HPF molecules on surfaces. Here we point out that the energy of probe light is negligible compared with the binding energies of reported $\mathrm{Ti} / \mathrm{CH}$ films being in the range from $280 \mathrm{eV}$ to $535 \mathrm{eV}$ [37].

For the convenience to compare dielectric constant and refractive index we have included the locus of dielectric line shown in Figure 2 in complex refractive index plane, which is now the parabola $n=-\kappa+\sqrt{\kappa^{2}\left(1+a^{2}\right)-a b}$ as shown in Figure 4. The knowledge of the parabola shaped locus in $(n$, $\kappa)$-plane (or linear shaped locus in $\left(\varepsilon^{\prime}, \varepsilon^{\prime \prime}\right)$-plane) helps us now to search the valid surface candidates, which are effective to adsorb HPF molecules without complicated experiments in vitro.

\section{Conclusion}

In the progress of this work we have noted the relation to surface parameters, which explain the ability of adsorption/desorption of HPF molecules (fraction I, type III) on the biosurfaces with different surface treatments. Three of the surfaces were titanium carbide surfaces performed utilizing plasma-enhanced chemical vapor deposition. The polished titanium and diamond-like carbon were acting as the reference surfaces.

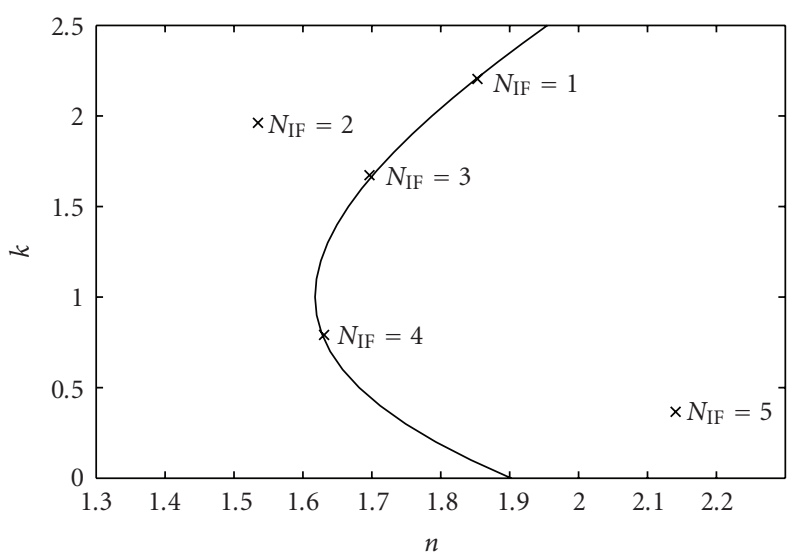

FIgURE 4: Complex effective refractive index $N_{n \kappa}=n+i \kappa$ for the five-treated titanium surfaces shown in Figure 3 at $E_{\lambda}=1.959 \mathrm{eV}$; whereas parabola $n=-\kappa+\sqrt{\kappa^{2}\left(1+a^{2}\right)-a b}$ with constants $a=$ 1.616 and $b=-5.848$ respects projection of dielectric line shown in Figure 2.

The significant correlation between the complex dielectric constant of dry titanium carbide surfaces and ability of adsorption of HPF molecules on these titanium carbide was observed, where permittivity $\varepsilon^{\prime}$ and dielectric loss $\varepsilon^{\prime \prime}$ have the known linear relation. Whereas the surface tension and surface energy of the titanium carbide samples, which was estimated from the optically measured contact angle of the droplet of distilled water (as well as the electrolyte droplet without and with HPF molecules) on the dry surface, did not give direct correlation with ability of adsorption of HPF on titanium carbide surfaces. The low correlation was also recognized from the surface capability to reflect energy back from the interface of air-titanium carbide surface. The findings in dielectric constants, which relate to interactions of the HPF molecules dissolved in the electrolyte and the titanium carbide surface, help us to understand mechanisms 
behind adsorption/desorption of HPF molecules on biomaterial surfaces in hard tissue replacement.

\section{Acknowledgments}

This work was supported by the Ministry of Education and Sport of the Czech Republic (1M0528), the Academy of Sciences of the Czech Republic (KAN200040651), the Grant Agency of the Czech Republic (202/08/1688, 205/10/2378), the Ministry of Education, Youth and Sports of the Czech Republic (LC06035), and institutional research plans, AVOZ50040507, AVOZ50040702).

\section{References}

[1] B. Walivaara, B.-O. Aronsson, M. Rodahl, J. Lausmaa, and P. Tengvall, "Titanium with different oxides: in vitro studies of protein adsorption and contact activation," Biomaterials, vol. 15 , no. 10, pp. 827-834, 1994.

[2] V. Ball, A. Bentaleb, J. Hemmerle, J.-C. Voegel, and P. Schaaf, "Dynamic aspects of protein adsorption onto titanium surfaces: mechanism of desorption into buffer and release in the presence of proteins in the bulk," Langmuir, vol. 12, no. 6, pp. 1614-1621, 1996.

[3] M. I. Jones, I. R. McColl, D. M. Grant, K. G. Parker, and T. L. Parker, "Protein adsorption and platelet attachment and activation, on TiN, TiC, and DLC coatings on titanium for cardiovascular applications," Journal of Biomedical Materials Research, vol. 52, no. 2, pp. 413-421, 2000.

[4] Y. Yang, R. Cavin, and J. L. Ong, "Protein adsorption on titanium surfaces and their effect on osteoblast attachment," Journal of Biomedical Materials Research Part A, vol. 67, no. 1, pp. 344-349, 2003.

[5] N. Huang, P. Yang, Y. X. Leng, et al., "Hemocompatibility of titanium oxide films," Biomaterials, vol. 24, no. 13, pp. 21772187, 2003.

[6] F. F Hook, J. Voros, M. Rodahl, et al., "A comparative study of protein adsorption on titanium oxide surfaces using in situ ellipsometry, optical waveguide lightmode spectroscopy, and quartz crystal microbalance/dissipation," Colloids and Surfaces $B$, vol. 24, no. 2, pp. 155-170, 2002.

[7] K. Imamura, M. Shimomura, S. Nagai, M. Akamatsu, and K. Nakanishi, "Adsorption characteristics of various proteins to a titanium surface," Journal of Bioscience and Bioengineering, vol. 106, no. 3, pp. 273-278, 2008.

[8] D. L. Cochran, "A comparison of endosseous dental implant surfaces," Journal of Periodontology, vol. 70, no. 12, pp. 15231539, 1999.

[9] T. J. Webster, R. W. Siegel, and R. Bizios, "Osteoblast adhesion on nanophase ceramics," Biomaterials, vol. 20, no. 13, pp. 1221-1227, 1999.

[10] P. M. Brett, J. Harle, V. Salih, et al., "Roughness response genes in osteoblasts," Bone, vol. 35, no. 1, pp. 124-133, 2004.

[11] E. Jansson and P. Tengvall, "Adsorption of albumin and IgG to porous and smooth titanium," Colloids and Surfaces B, vol. 35, no. 1, pp. 45-51, 2004.

[12] P. Cacciafesta, A. D. L. Humphris, K. D. Jandt, and M. J. Miles, "Human plasma fibrinogen adsorption on ultraflat titanium oxide surfaces studied with atomic force microscopy," Langmuir, vol. 16, no. 21, pp. 8167-8175, 2000.

[13] P. Cacciafesta, K. R. Hallam, A. C. Watkinson, G. C. Allen, M. J. Miles, and K. D. Jandt, "Visualisation of human plasma fibrinogen adsorbed on titanium implant surfaces with different roughness," Surface Science, vol. 491, no. 3, pp. 405$420,2001$.

[14] H. Nygren, P. Tengvall, and I. Lundstrom, "The initial reactions of $\mathrm{TiO}_{2}$ with blood," Journal of Biomedical Materials Research, vol. 34, no. 4, pp. 487-492, 1997.

[15] S. Kidoaki and T. Matsuda, "Adhesion forces of the blood plasma proteins on self-assembled monolayer surfaces of alkanethiolates with different functional groups measured by an atomic force microscope," Langmuir, vol. 15, no. 22, pp. 7639-7646, 1999.

[16] A. G. Hemmersam, M. Foss, J. Chevallier, and F. Besenbacher, "Adsorption of fibrinogen on tantalum oxide, titanium oxide and gold studied by the QCM-D technique," Colloids and Surfaces B, vol. 43, no. 3-4, pp. 208-215, 2005.

[17] M. Rouahi, E. Champion, O. Gallet, A. Jada, and K. Anselme, "Physico-chemical characteristics and protein adsorption potential of hydroxyapatite particles: influence on in vitro biocompatibility of ceramics after sintering," Colloids and Surfaces B, vol. 47, no. 1, pp. 10-19, 2006.

[18] R. Silvennoinen, K.-E. Peiponen, and K. Myller, Specular Gloss, Elsevier, Amsterdam, The Netherlands, 1st edition, 2008.

[19] R. Silvennoinen, V. Vetterl, S. Hasoň, et al., "Sensing of human plasma fibrinogen on polished, chemically etched and carbon treated titanium surfaces by diffractive optical element based sensor," Optics Express, vol. 16, no. 14, pp. 10130-10140, 2008.

[20] T. Vitu, T. Polcar, L. Cvrcek, et al., "Structure and tribology of biocompatible Ti-C:H coatings," Surface and Coatings Technology, vol. 202, no. 22-23, pp. 5790-5793, 2008.

[21] T. Polcar, T. Vitu, L. Cvrcek, R. Novak, J. Vyskocil, and A. Cavaleiro, "Tribological behaviour of nanostructured Ti-C:H coatings for biomedical applications," Solid State Sciences, vol. 11, no. 10, pp. 1757-1761, 2009.

[22] R. A. M. Azzam and N. M. Bashra, Ellipsometry and Polarized Light, North-Holland, Amsterdam, The Netherlands, 1977.

[23] S.-M. F. Nee and T.-W. Nee, "Principal Mueller matrix of reflection and scattering measured for a one-dimensional rough surface," Optical Engineering, vol. 41, no. 5, pp. 9941001, 2002.

[24] J. T. Räsänen, M. Savolainen, R. Silvennoinen, and K.-E. Peiponen, "Optical sensing of surface roughness and waviness by a computer-generated hologram," Optical Engineering, vol. 34, no. 9, pp. 2574-2580, 1995.

[25] P. Beckmann and A. Spizzichino, The Scattering of Electromagnetic Waves from Rough Surface, Pergamon Press, Oxford, UK, 1963.

[26] S. Donati, "Coupling dynamics in lasers and applications in self-mixing interferometry," in Proceedings of the 2nd International Meeting on Optical Sensing and Artificial Vision (OSAV'08), p. 67, 2008.

[27] O. V. Angelsky and P. P. Maksimyak, "Optical diagnostics of random phase objects," Applied Optics, vol. 29, pp. 2894-2898, 1990.

[28] O. V. Angelsky, A. P. Maksimyak, P. P. Maksimyak, and S. G. Hanson, "Optical correlation diagnostics of rough surfaces with large surface inhomogeneities," Optics Express, vol. 14, no. 16, pp. 7299-7311, 2006.

[29] R. M. Slawson, M. I. Van Dyke, H. Lee, and J. T. Trevors, "Germanium and silver resistance, accumulation, and toxicity in microorganisms," Plasmid, vol. 27, no. 1, pp. 72-79, 1992.

[30] I. Chopra, "The increasing use of silver-based products as antimicrobial agents: a useful development or a cause for concern?" Journal of Antimicrobial Chemotherapy, vol. 59, no. 4, pp. 587-590, 2007. 
[31] O. Akhavan and E. Ghaderi, "Enhancement of antibacterial properties of Ag nanorods by electric field," Science and Technology of Advanced Materials, vol. 10, no. 1, Article ID 015003, 2009.

[32] E. D. Palik, Ed., Handbook of Optical Constants of Solids, Volume I, II, and III, Academic Press, San Diego, Calif, USA, 1998.

[33] J.-M. Zhang, F. Ma, K.-W. Xu, and X.-T. Xin, "Anisotropy analysis of the surface energy of diamond cubic crystals," Surface and Interface Analysis, vol. 35, no. 10, pp. 805-809, 2003.

[34] S. Bar-Chaput and C. Carrot, "Interactions of active carbon with low- and high-molecular weight polyethylene glycol and polyethylene oxide," Journal of Applied Polymer Science, vol. 100, no. 5, pp. 3490-3497, 2006.

[35] W. Brandl, G. Marginean, V. Chirila, and W. Warschewski, "Production and characterisation of vapour grown carbon fiber/polypropylene composites," Carbon, vol. 42, no. 1, pp. 59, 2004.

[36] X. H. Zhang, A. Quinn, and W. A. Ducker, "Nanobubbles at the interface between water and a hydrophobic solid," Langmuir, vol. 24, no. 9, pp. 4756-4764, 2008.

[37] A. Grinevich, L. Bacakova, A. Choukourov, et al., "Nanocomposite $\mathrm{Ti} /$ hydrocarbon plasma polymer films from reactive magnetron sputtering as growth support for osteoblast-like and endothelial cells," Journal of Biomedical Materials Research Part A, vol. 88, no. 4, pp. 952-966, 2009. 

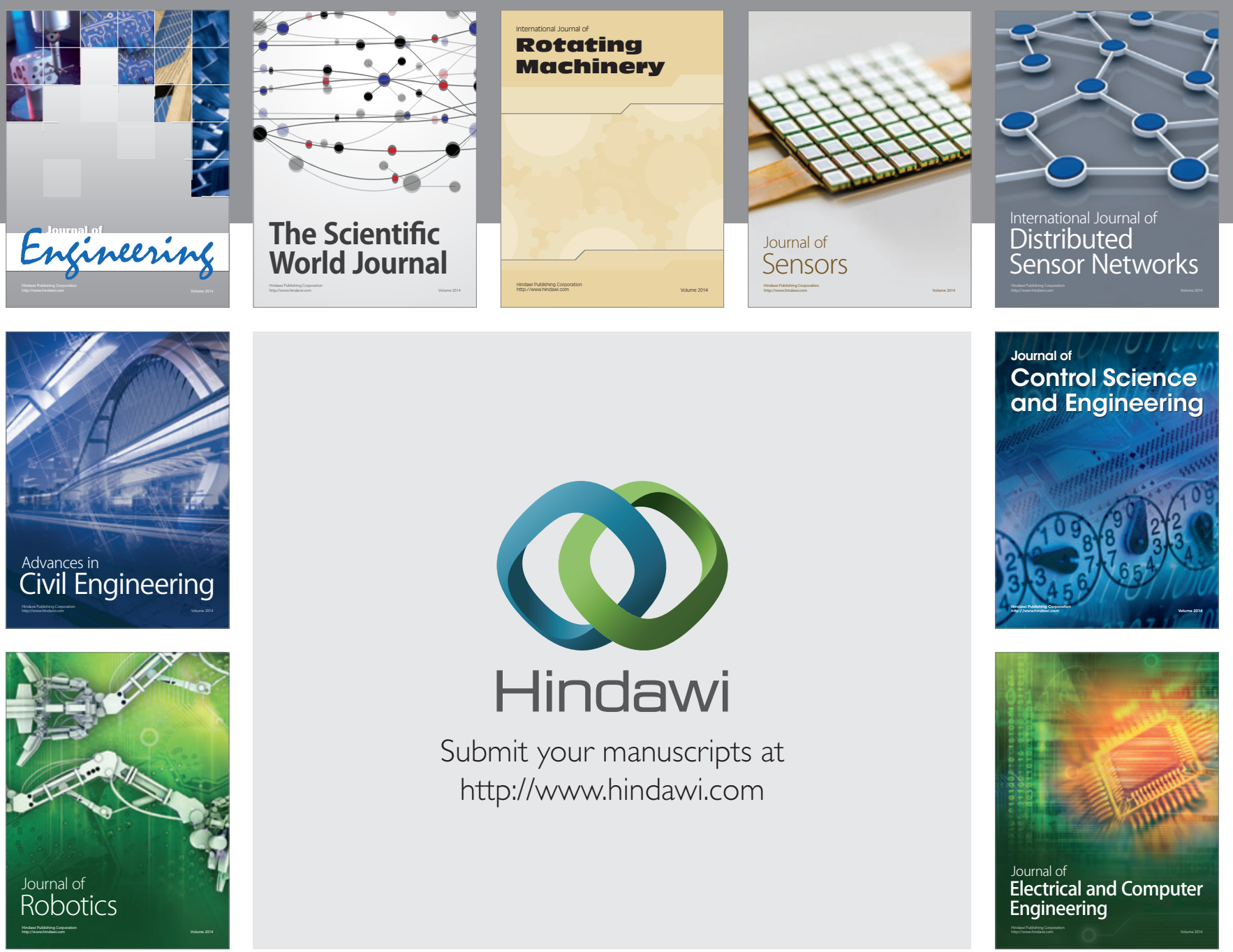

Submit your manuscripts at

http://www.hindawi.com
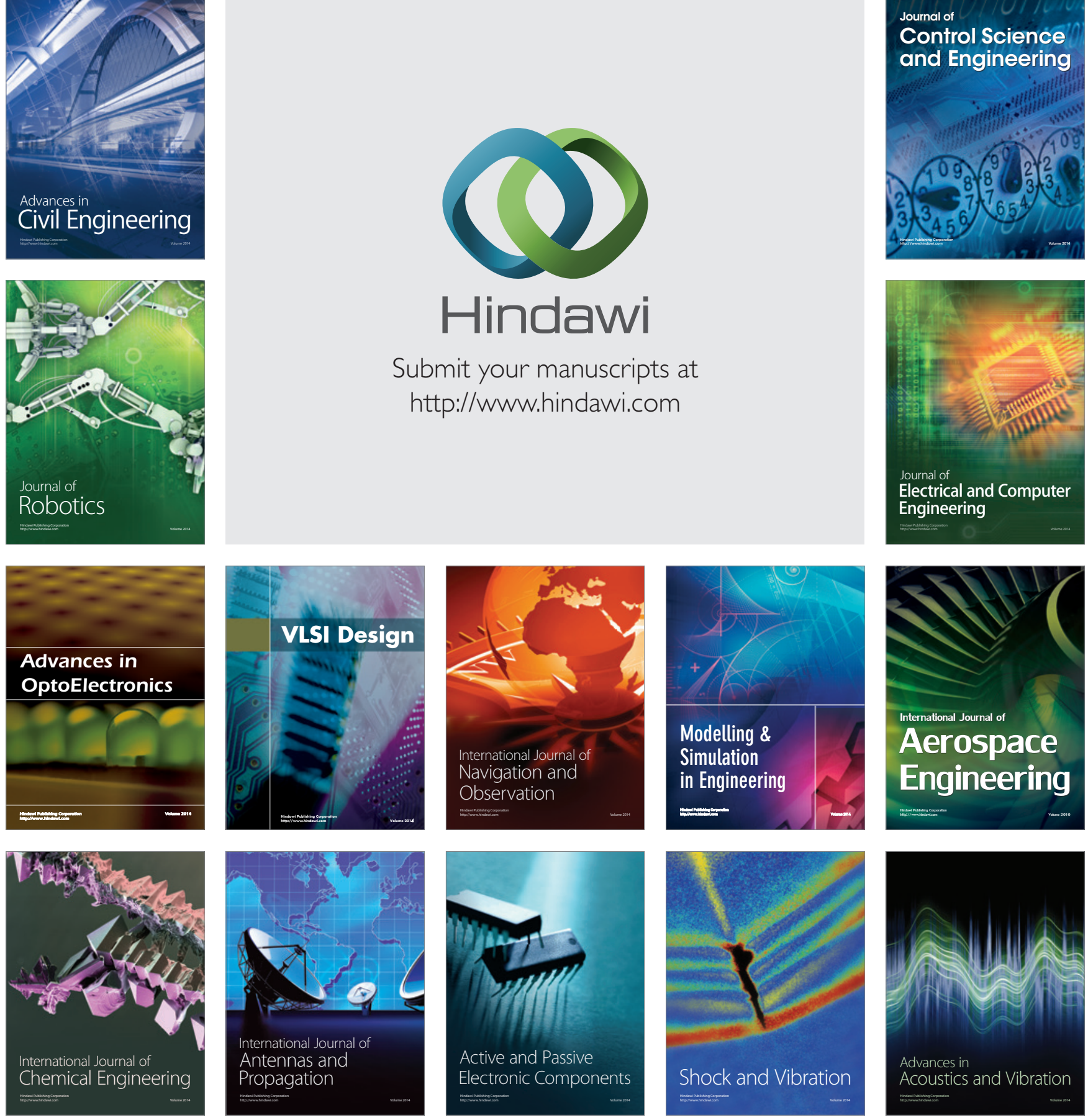